HUMBERTO FABIO BOATTO ${ }^{1}$ Maria Sayonara de Moraes ${ }^{2}$ Alexandre Paulo Machado ${ }^{3}$ Manoel joão Batista Castelo Girão ${ }^{4}$ OLGA FISCHMAN 5

\section{Artigos originais}

Palavras-chaves

Candidíase vulvovagina Candida albicans Técnicas e procedimentos de laboratório

Parceiros sexuais

Sinais e sintomas

Keywords

Candidiasis, vulvovagina Candida albicans Laboratory techniques and procedures Sexual partners Signs and symptoms
Correlação entre os resultados laboratoriais e os sinais e sintomas clínicos das pacientes com candidíase vulvovaginal e relevância dos parceiros sexuais na manutenção da infecção em São Paulo, Brasil

\author{
Relationship of laboratory results with clinical signs and symptoms \\ of patients with vulvovaginal candidiasis and the significance of the \\ sexual partners for the maintenance of the infection
}

\title{
Resumo
}

OBJETIVO: relacionar as leveduras identificadas aos sinais e sintomas clínicos das pacientes com candidíase vulvovaginal e investigar a importância dos parceiros sexuais na reincidência da infecção. MÉTODOS: foi desenvolvido estudo prospectivo de julho de 2001 a julho de 2003 com uma amostra de mulheres residentes na Grande São Paulo. Foram avaliadas 179 pacientes com suspeita clínica de vaginite fúngica, com idade entre 18 e 65 anos. Os critérios para exclusão foram: gravidez, comprometimento imunológico intrínseco e extrínseco, incluindo AIDS, diabetes, imunossupressão, pacientes em terapia com corticosteróides, antibióticos ou hormônios, em pós-menopausa, em uso de dispositivo intrauterino e duchas vaginais ou espermicidas. Amostras de secreções vaginais ou da glande dos parceiros sexuais de pacientes com vaginite de repetição foram coletadas para microscopia e cultura de fungos. Colônias fúngicas isoladas em CHROMagar Candida foram identificadas por provas clássicas. O teste exato de Fisher foi usado para correlacionar o quadro clínico com as leveduras isoladas das pacientes. RESULTADOS: os sinais e sintomas clínicos mais relevantes na candidíase vulvovaginal foram prurido e corrimento, seguidos por eritema e edema, estatisticamente independente do agente etiológico. Leveduras foram diagnosticadas por microscopia direta em 77 pacientes com vulvovaginites, sendo obtidos 40 cultivos de Candida spp. Candida albicans (70\%), C. glabrata (20\%), C. tropicalis (7,5\%) e C. guilliermondii $(2,5 \%)$ foram identificadas. As leveduras prevalentes nos parceiros foram C. albicans e C. glabrata. As mesmas espécies foram detectadas nas companheiras e parceiros em $87 \%$ dos casos. CONCLUSÕES: as vulvovaginites fúngicas foram mais freqüentes em mulheres entre 18 e 34 anos de idade. Não foi observada correlação entre as espécies de leveduras detectadas e a sintomatologia clínica. Os parceiros sexuais podem ser importantes reservatórios de Candida spp e estar relacionados à manutenção da candidíase vulvovaginal.

\section{Abstract}

PURPOSE: to relate yeasts identified by laboratory tests to clinical signs and symptoms in patients with vulvovaginal candidiasis, and to investigate the importance of the sexual partners in the recurrence of the infection. METHODS: from July 2001 to July 2003, a sample of 179 patients aged from 18 to 65 years old, with clinical suspicion of fungal vaginitis were analyzed in a prospective study in Great São Paulo. Exclusion criteria included: pregnancy, impaired intrinsic or extrinsic immune response (including Aids), diabetes or immunosuppression; patients undergoing corticosteroid, antibiotic or hormone therapy, in post menopause, with intrauterine device (IUD) or making use of vaginal douches or spemicides. Samples of vaginal and penis secretions from partners of patients with relapse of vaginitis episodes were collected for microscopy and fungal culture. Fungal colonies isolated in CHROMagar Candida were identified by classical methods. Fisher's exact ttest was used to correlate the clinical picture with the yeasts isolated from patients. RESULTS: the most relevant clinical signs and symptoms were pruritus and vaginal discharge followed by erythema and edema, statistically independent from the etiological agent. Direct microscopy revealed yeasts in 77 patients with vulvovaginitis, and 40 Candida spp cultures were obtained. Candida albicans (70\%), C. glabrata (20\%), C. tropicalis (7,5\%) and C. guilliermondii (2,5\%) were identified. The yeasts prevalent in partners were C. albicans and C. glabrata. The same species were detected in

Correspondência:

Olga Fischman Departamento de Microbiologio Imunologia e Parasitologia da EPM/UNIFESP Rua Botucatu, 862,80 andar - Edifício Ciências Biomédicas CEP 04023-062 - São Paulo/SP Fone: (11) 5576-4523, ramal 15 E-mail: olga@ecb.epm.br
Trabalho realizado no Departamento de Microbiologia, Imunologia e Parasitologia, Disciplina de Biologia Celular da Universidade Federal de São Paulo - UNIFESP.

Ginecologista da Unidade Básica de Saúde CS-II de Jardim Castelo de Ferraz de Vasconcelos - São Paulo (SP), Brasil. 2 Dermatologista da Unidade Básica de Saúde CS-ll de Jardim Castelo de Ferraz de Vasconcelos - São Paulo (SP), Brasil. 3 Professor Assistente de Microbiologia da Universidade Federal do Mato Grosso - UFMT - Mato Grosso (MT), Brasil.

${ }^{4}$ Chefe do Departamento de Ginecologia da Escola Paulista de Medicina da Universidade Federal de São Paulo - UNIFESP São Paulo (SP), Brasil.

5 Professora Adjunta do Departamento de Microbiologia, Imunologia e Parasitologia da Escola Paulista de Medicina da Universidade Federal de São Paulo - UNIFESP - São Paulo (SP), Brasil. 
female and male sex partners in $87 \%$ of the cases. CONCLUSIONS: fungal vulvovaginitis was more frequent in women between 18 and 34 years old

No correlation was observed between the species of yeast detected and clinical symptomatology. Sexual partners are important Candida spp reservoirs and may be related to the maintenance of the vulvovaginal candidiasis.

\section{Introdução}

As vaginites infecciosas são causadas, principalmente, por bactérias, fungos leveduriformes e Trichomonas vaginalis ${ }^{1}$. Leveduras podem colonizar as mucosas ou estar associadas a quadros de vulvovaginites, especialmente Candida spp. Estes microrganismos são detectados em cerca de $10 \%$ das mulheres em pré-menopausa, 5 a $10 \%$ na menopausa e $30 \%$ das gestantes, sem necessariamente terem ligação com quadros de vaginites fúngicas ${ }^{2,3}$. Candidíase vulvovaginal é um processo infeccioso e/ou inflamatório do trato geniturinário inferior feminino relevante na medicina devido ao acentuado número de atendimentos em consultórios de ginecologia ${ }^{3-5}$. Prurido, leucorréia, placas esbranquiçadas, edema e eritema na vulva e vagina têm sido as manifestações clínicas mais comuns nesse tipo de infecção ${ }^{2,3,5-8}$. A sintomatologia das vulvovaginites fúngicas não é patognomônica e a suspeita clínica pode ser confirmada por testes que evidenciam a natureza do agente etiológico ${ }^{8}$. Por essa razão, os exames laboratoriais devem ser realizados para o diagnóstico diferencial de outras vaginites infecciosas, resultando em benefícios aos pacientes pelos tratamentos específicos, que evitam o agravamento da infecção, procedimentos médicos e custos desnecessários ${ }^{2,3,8}$. Um outro fator relevante tem sido a detecção de resistência in vitro de Candida spp aos antifúngicos comumente empregados na prática clínica e o aumento progressivo do isolamento de espécies não-albicans, como C. tropicalis, C. krusei, C. glabrata e $C$. guilliermondi $i^{9-12}$, ainda que $C$. albicans seja o microrganismo diagnosticado em mais de $70 \%$ das ocorrências de candidíase vulvovaginal ${ }^{3-5,13,14}$. As leveduras, em geral, são caracterizadas por métodos clássicos, como análise da micromorfologia e do perfil bioquímico ${ }^{2,3}$. Atualmente, meios cromogênicos têm sido empregados na identificação presuntiva de algumas espécies, o que torna o diagnóstico mais rápido e possibilita a distinção de mais de um agente em cultura ${ }^{15-17}$.

Embora o consenso sobre candidíase vulvovaginal recorrente não seja bem estabelecido, a condição de recorrência é definida quando são diagnosticados pelo menos quatro episódios durante um ano, enquanto que as vaginites de repetição ocorrem esporadicamente, com três ou menos episódios ao ano ${ }^{18,19}$. A transmissão sexual tem sido citada como fator importante na reincidência da doença $a^{5,18-20}$.

$\mathrm{Na}$ presente pesquisa, procuramos estudar as manifestações clínicas das vulvovaginites fúngicas, investigando a correlação com as espécies de leveduras isoladas e a condição dos parceiros sexuais como reservatórios de Candida spp nos casos de vaginites de repetição.

\section{Métodos}

Entre julho de 2001 e julho de 2003 , foram atendidas 1.825 pacientes em ambulatórios de Ginecologia de Postos de Saúde nos municípios de Poá, Ferraz de Vasconcelos e Mogi das Cruzes (Grande São Paulo, Brasil). Das 423 pacientes com diagnóstico de vulvovaginites, 179 que tinham suspeita clínica de vaginites fúngicas por apresentarem uma ou mais manifestações como leucorréria, prurido, disúria, edema, eritema, queimação/ardência e dor vulvar, foram examinadas em estudo prospectivo. Os sinais e sintomas foram anotados em ficha individual e receberam pontuações arbitradas conforme a seguinte escala: $0=$ ausente, $1=$ leve, $2=$ moderado e $3=$ severo. Foram consideradas pacientes com quadros clínicos leves as que obtiveram de 0 a 6 pontos; moderados, de 7 a 13; e severos, mais de 13 pontos.

As pacientes foram informadas sobre o protocolo e, após a concordância voluntária sobre sua participação no estudo, assinaram o termo de consentimento. O projeto de pesquisa foi aprovado pelo comitê de Ética em Pesquisa da Universidade Federal de São Paulo (UNIFESP) do Hospital São Paulo, sob nº 1083/01.

Foram excluídas da investigação pacientes com menos de 18 anos ou com idade superior a 65 anos, as grávidas, com comprometimento imunológico intrínseco ou adquirido, incluindo HIV +, diabéticas, submetidas à terapia de corticosteróides, antibióticos e/ou hormônios, em uso de dispositivo intrauterino e duchas vaginais espermicidas. A distribuição por idade foi a seguinte: de 18 a 24 anos: 56, de 25 a 29: 50, de 30 a 34: 29, de 35 a 39: 20, de 40 a 44: 13, de 45 a 49: 8, de 50 a 54: 2 e de 60 a 65: 1. Entre as pacientes estudas, sete com suspeita de candidíase vulvovaginal com idade superior a 44 anos estavam na menopausa.

As secreções vaginais da ectocérvix e do fórnice vaginal, bem como secreções do prepúcio e da glande de 58 parceiros sexuais, coletadas com swab umedecido em solução fisiológica esterilizada, foram submetidas à microscopia direta com KOH $10 \%$ e os esfregaços corados pelo método de Gram.

As amostras foram semeadas em meio de CHROMagar Candida e repicadas, posteriormente, em ágar Sabouraud dextrose. A identificação dos isolamentos foi 
feita pelos métodos clássicos, compreendendo análise da micromorfologia e perfil bioquímico e fisiológico. Testes de assimilação e fermentação de carboidratos foram realizados para todos os isolados em duplicata. A caracterização definitiva de $C$. albicans foi obtida pela visualização microscópica de clamidoconídios, após cultivo dos isolados em ágar fubá-Tween 80 à temperatura ambiente, durante 72 horas, e produção de tubo germinativo, em soro de coelho, mantido a $37^{\circ} \mathrm{C}$, durante

Tabela 1 - Distribuição da idade das pacientes com suspeita de candidíase vulvovaginal pelos resultados dos exames laboratoriais.

\begin{tabular}{c|c|c|cc}
\hline Idade & Exames (-) & Ex. dir. (+) Cultura (-) & Ex. dir. (+) Cultura (+) & Total \\
\hline $18-24$ & 35 & 15 & 6 & 56 \\
$25-29$ & 27 & 12 & 11 & 50 \\
$30-34$ & 16 & 2 & 11 & 29 \\
$35-39$ & 15 & 2 & 3 & 20 \\
$40-44$ & 9 & - & 4 & 13 \\
$45-49$ & - & 5 & 3 & 8 \\
$50-54$ & - & 1 & 1 & 2 \\
$55-59$ & - & - & - & - \\
$60-65$ & - & - & 1 & 1 \\
\hline Total & 102 & 37 & 40 & 179 \\
\hline
\end{tabular}

Ex. Dir. : Exame micológico direto; $(-)=$ negativo; $(+)=$ positivo.

Tabela 2 - Espécies de leveduras isoladas de acordo com a intensidade do quadro clínico.

\begin{tabular}{lcccc}
\hline Espécies & Leve & Moderado* & Severo & Total \\
\hline Candida albicans & 8 & 20 & - & 28 \\
Candida glabrafa & 2 & 6 & - & 8 \\
Candida tropicalis & - & 2 & 1 & 3 \\
Candida guilliermondii & - & 1 & - & 1 \\
\hline Total & 10 & 29 & 1 & 40 \\
\hline
\end{tabular}

*Quadro clínico moderado foi observado entre a maior parte das pacientes $(72,5 \%)$; o teste exato de Fisher (intensidade do quadro clínico versus espécies isoladas) não foi significante $(p=0,726)$.

Tabela 3 - Espécies de leveduras isoladas de casos de vaginites e de vaginites de repetição.

\begin{tabular}{lccccccc}
\hline \multirow{2}{*}{ Espécies } & \multicolumn{2}{c}{ Vaginites } & \multicolumn{2}{c}{ Vaginifes de repeticã̃ } & \multicolumn{2}{c}{ Total } \\
\cline { 2 - 8 } & $\mathbf{n}$ & $\%$ & $\mathrm{n}$ & $\%$ & $\mathrm{n}$ & $\%$ \\
\hline Candida albicans & 13 & 32,5 & 15 & 37,5 & 28 & 70 \\
Candida glabrafa & 1 & 2,5 & 7 & 17,5 & 8 & 20 \\
Candida tropicalis & 2 & 5 & 1 & 2,5 & 3 & 7,5 \\
Candida guilliermondii & - & - & 1 & 2,5 & 1 & 2,5 \\
\hline Tofal & 16 & 40 & 24 & 60 & 40 & 100 \\
\hline
\end{tabular}

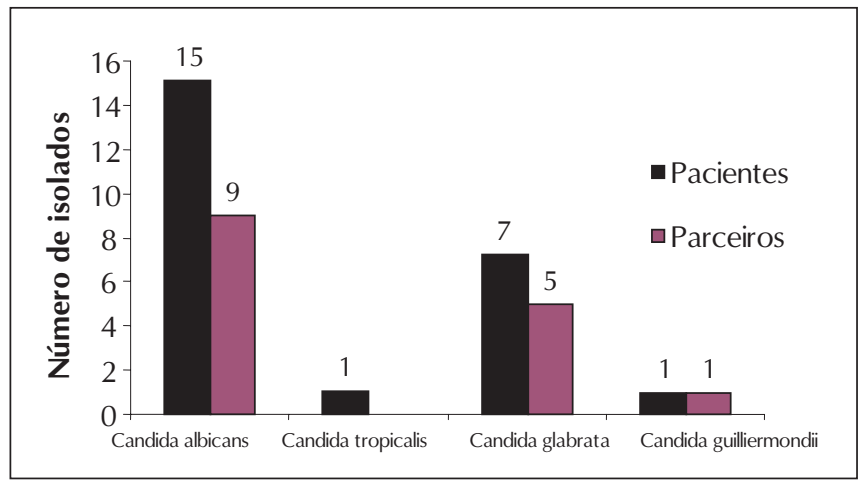

Figura 1 - Leveduras identificadas nas pacientes e em seus parceiros. duas horas. O teste exato de Fisher foi usado para verificar a correlação dos quadros clínicos/intensidades, sinais e sintomas com as diferentes espécies de Candida isoladas. Pacientes com cultivo positivo foram acompanhadas por exame clínico e laboratorial, mensalmente, durante 12 meses, a fim de verificar repetição ou recorrência, sem terem sido consideradas como novos casos.

\section{Resultados}

As manifestações de vulvovaginites foram mais prevalentes em mulheres de 18 a 34 anos. Em 102 (57\%) dos 179 casos com diagnóstico clínico de vulvovaginite fúngica não foi verificada a presença de leveduras (Tabela 1). A suspeita clínica foi confirmada por exame microscópico em 77 pacientes (43\%), sendo sete na menopausa. $\mathrm{O}$ exame microscópico direto do material corado pelo método de Gram e clarificado com $\mathrm{KOH}$ $10 \%$ demonstrou a mesma proporção de positividade, embora as estruturas fúngicas fossem melhor visualizadas quando coradas pelo método citado.

Foram identificados 40 isolamentos de Candida spp. As espécies isoladas foram C. albicans (70\%), C. glabrata (20\%), C. tropicalis $(7,5 \%)$ e C. guilliermondii $(2,5 \%)$. Todos os isolados de C. albicans e C. tropicalis apresentaram coloração típica, verde e azul, respectivamente, em CHROMagar Candida. Três espécies, $C$ albicans, $C$. tropicalis e C. glabrata, foram isoladas de uma paciente em meio cromogênico.

Os sinais e sintomas clínicos registrados e manifestados com maior ou menor intensidade foram prurido, corrimento, disúria, eritema e ardência, com predominância de corrimento e prurido, quase sempre persistentes nos diversos episódios. De acordo com a escala aplicada, os sinais clínicos foram considerados moderados em 29 casos, leves em dez casos e severos em apenas uma ocorrência. Não houve correlação estatística entre a intensidade dos sinais e sintomas observados e as espécies de leveduras identificadas (Tabela 2).

Vulvovaginites de repetição foram observadas em 24 $(60 \%)$ mulheres (Tabela 3), das quais 16 apresentaram dois episódios; seis, três episódios e duas, quatro episódios, com tempo médio de 92,7 dias entre os episódios. Dos 58 companheiros sexuais examinados, 38 eram sintomáticos e 20 assintomáticos. Cultivos foram obtidos de cinco parceiros sintomáticos: C. albicans $(\mathrm{n}=3)$ e $C$. glabatra $(\mathrm{n}=2)$ e de dez assintomáticos: $C$. albicans $(\mathrm{n}=6), C$. glabatra $(\mathrm{n}=3)$ e C. guilliermondii $(\mathrm{n}=1)$. Desses 15 cultivos de Candida spp obtidos a partir das secreções da glande e prepúcio, $13(87 \%)$ apresentaram correspondência com espécies isoladas das respectivas companheiras (Figura 1). 


\section{Discussão}

As vulvovaginites são responsáveis por cerca de 20 a 30\% das doenças ginecológicas observadas em mulheres de 20 a 30 anos de idade ${ }^{13}$, sendo a candidíase vulvovaginal a segunda causa dessa infecção ${ }^{14}$. Estima-se que $75 \%$ das mulheres terão, ao menos, um episódio de vaginite fúngica durante sua vida reprodutiva, 40 a $50 \%$ teriam uma segunda infecção e, aproximadamente, $5 \%$ delas desenvolverão um curso crônico ${ }^{2,5,19}$. Em nossa investigação, as vaginites foram motivo de consulta de $23 \%$ das pacientes atendidas. A maior parte das mulheres com vaginites fúngicas tinha idade entre 18 e 34 anos e somente sete encontravam-se na menopausa, o que confirma a prevalência da candidíase na menacme ${ }^{8,14}$.

Prurido e corrimento têm sido as manifestações clínicas mais observadas nas vaginites fúngicas $2,3,5,7,8,21$. Corrimento branco ou branco-amarelado (100\%) e prurido $(97,5 \%)$ foram as queixas mais freqüentes, seguidas de eritema, edema, ardência e disúria, sendo mais acentuados no período pré-menstrual e pós-relações sexuais. Embora a percentagem de pacientes com os principais sinais e sintomas da candidíase vulvovaginal seja elevada, o diagnóstico clínico é apenas sugestivo, devendo ser confirmado por provas laboratoriais, pois manifestações similares podem ser causadas por outros agentes infecciosos ou por condições ligadas ao hospedeiro $^{2,3,8}$. Na presente pesquisa, a suspeita clínica de candidíase vulvovaginal não foi comprovada por exames laboratoriais em 102 casos (57\%).

A quantidade de leveduras aumenta expressivamente no processo infeccioso, o que facilita a sua detecção ${ }^{3}$. O exame microscópico das secreções vaginais coradas ao Gram ou clarificadas com $\mathrm{KOH}$ mostrou resultados idênticos. Entretanto, a coloração dos esfregaços pelo método de Gram é interessante para o diagnóstico de vaginites fúngicas, pois demonstra melhor as estruturas invasivas das leveduras ${ }^{22}$. A obtenção de 40 cultivos ( $52 \%$ das amostras positivas por microscopia) foi menos sensível que o exame direto. Esse fato pode ser atribuído à escassez ou inadequabilidade do material biológico colhido em determinados sítios da vulva ou vagina, quantidade insuficiente de leveduras no espécime clínico para o isolamento ou baixa viabilidade dos microrganismos devido ao uso prévio de automedicação sem comunicação ao clínico. Todavia, a combinação entre exame direto e cultura é ideal para o diagnóstico das vaginites fúngicas ${ }^{22}$.

O meio de cultura mais usado na rotina micológica para cultivo dos fungos patogênicos é o ágar Sabouraud dextrose. Todavia, o isolamento de diferentes espécies de Candida de um mesmo material clínico é difícil, uma vez que as características morfológicas das colônias são muito semelhantes. CHROMagar Candida é um meio de cultura cromogênico que permite a diferenciação presuntiva de determinadas espécies de leveduras, particularmente das mais prevalentes em vulvovaginites fúngicas, sendo recomendável no diagnóstico de culturas mistas, o que melhora a qualidade dos exames laboratoriais ${ }^{15-17}$. A utilização desse meio permitiu o isolamento de três espécies diferentes (C.albicans, $C$. tropicalis e $C$. glabrata) de uma mesma paciente, o que não é evento comum. $C$. albicans $(70 \%)$ e C. glabrata (20\%) foram os microrganismos mais isolados, mostrando boa correlação com os dados de outros pesquisadores, que identificaram C. albicans em 70,1\% e C. glabrata em $18,9 \%$ dos $\operatorname{casos}^{12}$. Todavia, índices mais elevados de C. albicans, de 80 a $90 \%$, têm sido descri$\operatorname{tos}^{10,13,16,22}$. C. dubliniensis, levedura emergente, tem sido isolada em percentual bastante reduzido $(0,17 \%)$ entre isolados vaginais de C. albicans ${ }^{23}$. Candida tropicalis (7,5\%) e C. guilliermondii (2,5\%) foram também diagnosticadas, não sendo encontradas $C$. parapsilosis e $C$. krusei, espécies isoladas em outros estudos ${ }^{7,12,16,21}$. Ausência de correlação entre sinais/sintomas e cultura positiva foi demonstrada em um estudo transversal ${ }^{8}$. Em concordância, em nossa investigação não foi estabelecida relação entre sintomatologia e espécies de leveduras isoladas.

Dos 40 casos de vaginite fúngica em que o agente foi identificado, 24 tiveram vaginite de repetição. Leveduras presentes no trato intestinal no períneo e no parceiro sexual podem estar relacionadas às vaginites de repetição ${ }^{3,8,18,19}$. Verificamos que em $87 \%$ dos casos a mesma espécie de levedura estava presente na mulher e em seu companheiro. Entretanto, inúmeros fatores ainda desconhecidos, associados ao desenvolvimento da candidíase vulvovaginal e da sua recorrência, necessitam ser esclarecidos. A comparação dos isolados do períneo feminino, dos parceiros sexuais e dos diferentes episódios das vaginites fúngicas por técnicas de filogenia molecular permitirá, num futuro próximo, uma maior compreensão da epidemiologia da candidíase vulvovaginal.

Nesse estudo, concluiu-se que sinais e sintomas clínicos, predominantemente moderados, como prurido, corrimento e outros menos prevalentes, são presuntivos e não se correlacionaram com as espécies isoladas. Assim, os testes laboratoriais são importantes para confirmação etiológica do agente, inclusive de infecções mistas. Em casos de reinfecção, os parceiros sexuais devem ser avaliados devido à possibilidade de serem reservatórios de Candida spp.

\section{Agradecimentos}

Agradecimentos ao $\mathrm{CNPq}$ pelo apoio financeiro. A André Martins pela tipagem dos isolados. 


\section{Referências}

1. Geva A, Bornstein J, Dan M, Shoham HK, Sobel JD. The VI-SENSEvaginal discharge self-test to facilitate management of vaginal symptoms. Am J Obstet Gynecol. 2006; 195(5):1351-6.

2. Schaller M. [Candida albicans - interactions with the mucosa and the immune system]. J Dtsch Dermatol Ges. 2006; 4(4):328-36; quiz 337-8. German.

3. Mendling W, Seebacher C, Working Group for Infections and Infectimmunology of the German Society for Gynecology and Obstetrics; German Speaking Mycological Society; German Dermatological Society. Guideline vulvovaginal candidosis: guideline of the German Dermatological Society, the German Speaking Mycological Society and the Working Group for Infections and Infectimmunology of the German Society for Gynecology and Obstetrics. Mycoses. 2003; 46(9-10):365-9.

4. Barrenetxea Ziarrusta G. Vulvovaginitis candidiásica. Rev lberoam Micol. 2002; 19(1):22-4.

5. Ferrer J. Vaginal candidosis: epidemiological and etiological factors. Int J Gynaecol Obstet. 2000; 71 Suppl 1:S21-7.

6. Odds FC, Webster CE, Mayuranathan P, Simmons PD. Candida concentrations in the vagina and their association with signs and symptoms of vaginal candidosis. J Med Vet Mycol. 1988; 26(5):277-83.

7. Lopes Consolaro ME, Aline Albertoni T, Shizue Yoshida C, Mazucheli J, Peralta RM, Estivalet Svidzinski TI. Correlation of Candida species and symptoms among patients with vulvovaginal candidiasis in Maringa, Parana, Brazil. Rev Iberoam Micol. 2004; $21(4): 202-5$

8. Rosa MI, Rumel D. Fatores associados à candidíase vulvovaginal: estudo exploratório. Rev Bras Ginecol Obstet. 2004; 26(1): 65-70.

9. Spinillo A, Capuzzo E, Gulminetti R, Marone P, Colonna L, Piazzi $G$. Prevalence of and risk factors for fungal vaginitis caused by non-albicans species. Am J Obstet Gynecol. 1997; 17611 Pt 1):138-41

10. Ribeiro MA, Dietze R, Paula CR, Da Matta DA, Colombo AL. Susceptibility profile of vaginal yeast isolates from Brazil. Mycopathologia. 2001; 151(1):5-10.
11. Crocco El, Mimica LM, Muramatu LH, Garcia C, Souza VM, Ruiz LRB, et al. Identificação de espécies de Candida e susceptibilidade antifúngica in vitro: estudo de 100 pacientes com candidíases superficiais. An Bras Dermatol. 2004; 79(6):689-97.

12. Richter SS, Galask RP, Messer SA, Hollis RJ, Diekema DJ, Pfaller MA. Antifungal susceptibilities of Candida species causing vulvovaginitis and epidemiology of recurrent cases. J Clin Microbiol. 2005; 43(5):2155-62.

13. Azzam-W M, Cermeno-Vivas JR, Orellan-Garcia Y, Penna SJ. Vulvovaginitis caused by Candida spp. and Trichomonas vaginalis in sexually active women. Invest Clin. 2002; 43(1):3-13.

14. Galle LC, Gianinni MSM. Prevalência e susceptibilidade de leveduras vaginais. J Bras Patol Med Lab. 2004; 40(4):229-36.

15. Willinger B, Manafi M. Evaluation of CHROMagar Candida for rapid screening of clinical specimens for Candida species. Mycoses. 1999; 42(1-2):61-5.

16. Novikova N, Rodrigues A, Mardh PA. Can the diagnosis of recurrent vulvovaginal candidosis be improved by use of vaginal lavage samples and cultures on chromogenic agar? Infect Dis Obstet Gynecol. 2002; 10(2):89-92.

17. Gultekin B, Yazici V, Aydin N. Distribution of Candida species in vaginal specimens and evaluation of CHROMagar Candida medium. Mikrobiyol Bul. 2005; 39(3):319-24.

18. Ringdahl EN. Treatment of recurrent vulvovaginal candidiasis. Am Fam Physician. 2000; 61 (1 1):3306-12, 3317

19. Fidel PL Jr, Sobel JD. Immunopathogenesis of recurrent vulvovaginal candidiasis. Clin Microbiol Rev. 1996; 9(3):335-48.

20. Sobel JD. Pathogenesis and treatment of recurrent vulvovaginal candidiasis. Clin Infect Dis. 1992; 14 Suppl 1:S148-53.

21. Moreira D, Paula CR. Vulvovaginal candidiasis. Int J Gynaecol Obstet. 2006; 92(3):266-7.

22. Omar AA. Gram stain versus culture in the diagnosis of vulvovaginal candidiasis. East Mediterr Health J. 2001; 7(6):925-34.

23. Acikgoz ZC, Sancak B, Gamberzade S, Misirlioglu M. Prevalence of Candida dubliniensis among the stored vaginal Candida isolates in a Turkish hospital. Mycoses. 2004; 47(9-10):393-6. 